\title{
The IGS-ETS in Bacillus (Insecta Phasmida): molecular characterization and the relevance of sex in ribosomal DNA evolution
}

\author{
Andrea Ricci, Valerio Scali and Marco Passamonti*
}

Address: Dipartimento di Biologia Evoluzionistica Sperimentale, University of BolognaVia Selmi 3, 40126 Bologna, Italy

Email: Andrea Ricci - and.ricci@gmail.com; Valerio Scali - valerio.scali@unibo.it; Marco Passamonti* - marco.passamonti@unibo.it

* Corresponding author

Published: 9 October 2008

BMC Evolutionary Biology 2008, 8:278 doi:10.1/86//47/-2/48-8-278

This article is available from: http://www.biomedcentral.com/I47I-2/48/8/278

(c) 2008 Ricci et al; licensee BioMed Central Ltd.

This is an Open Access article distributed under the terms of the Creative Commons Attribution License (http://creativecommons.org/licenses/by/2.0), which permits unrestricted use, distribution, and reproduction in any medium, provided the original work is properly cited.

\begin{abstract}
Background: DNA encoding for ribosomal RNA (rDNA) is arranged in tandemly-repeated subunits, each containing ribosomal genes and non-coding spacers. Because tandemly-repeated, rDNA evolves under a balanced influence of selection and "concerted evolution", which homogenizes rDNA variants over the genome (through genomic turnover mechanisms) and the population (through sexuality).
\end{abstract}

Results: In this paper we analyzed the IGS-ETS of the automictic parthenogen Bacillus atticus and the bisexual B. grandii, two closely related stick-insect species. Both species share the same IGSETS structure and sequence, including a peculiar head-to-tail array of putative transcription enhancers, here named Bag530. Sequence variability of both IGS-ETS and Bag530 evidenced a neat geographic and subspecific clustering in $B$. grandii, while $B$. atticus shows a little but evident geographic structure. This was an unexpected result, since the parthenogen $B$. atticus should lack sequence fixation through sexuality. In $B$. atticus a new variant might spread in a given geographic area through colonization by an all-female clone, but we cannot discard the hypothesis that $B$. atticus was actually a bisexual taxon in that area at the time the new variant appeared. Moreover, a gene conversion event between two Bag530 variants of B. grandii benazzii and B. grandii maretimi suggested that rRNA might evolve according to the so-called "library hypothesis" model, through differential amplification of rDNA variants in different taxa.

Conclusion: On the whole, Bacillus rDNA evolution appears to be under a complex array of interacting mechanisms: homogenization may be achieved through genomic turnover that stabilizes DNA-binding protein interactions but, simultaneously, new sequence variants can be adopted, either by direct appearance of newly mutated repeats, or by competition among repeats, so that both DNA-binding proteins and repeat variants drive each other's evolution. All this, coupled with chromosome reshuffling due to sexuality (when present), might drive a quick fixation of new rDNA variants in the populations. 


\section{Background}

Ribosomal RNA genes (rDNA) encode the RNA scaffold of the ribosome and thereby perform what likely is the most basic of all housekeeping functions. Within the rDNA cluster, each repeat contains one copy of the 18S, 5.8S and $28 \mathrm{~S}$ rRNA genes, which are separated by several spacers: two internal transcribed spacers, ITS1 (between $18 \mathrm{~S}$ and 5.8S) and ITS2 (between 5.8S and 28S), an external transcribed spacer (ETS, between promoter and 18S), and an untranscribed intergenic spacer (IGS), separating each adjacent rDNA unit. The rDNA unit is transcribed by the RNA polymerase I (RNA-PolI) as a single $45 \mathrm{~S}$ precursor molecule, which is subsequently processed by an ordered cleavage of the spacers. The rDNA units are organized in large tandem arrays (rDNA loci), generally located within one or a few chromosome pairs, where they constitute the nucleolar organizer regions (NOR). During active synthesis, rDNA loci are associated to the nucleolus (for a review see [1]).

While the core domain of the rDNA coding region is well conserved, the IGS is rapidly evolving, so that it has been used as a marker for identification of closely related species and for quantification of gene flow between populations ([2] and references therein). In eukaryotes, tandemly repeated multigene families, like rDNA, undergo a peculiar pattern of evolution known as "concerted evolution" [3]: as a consequence of this, the repeats show a higher sequence homogeneity within a species (or population) than between species (or populations). This pattern is achieved through two independent mechanisms known as homogenization (i.e. the spreading of a variation to the whole repeat array) and fixation (i.e. the fixation of the variant within a taxonomic unit). Homogenization is achieved through several genomic turnover mechanisms, such as unequal crossing-over and gene conversion, while fixation is a by-product of the chromosome reshuffling due to sexual reproduction [3].

Despite the presence of molecular mechanisms that homogenize the ribosomal arrays, variations in rDNA have been commonly observed both within and among populations of several species. This variation is both quantitative and qualitative, the former consisting in a redundancy of rDNA copies and in the number of loci per genome, the latter being typically due to the occurrence of regulatory sub-repeats within the rDNA cluster [4]. Actually, the IGS contains the RNA-PolI promoter and other important regulatory elements, such as terminators, spacer promoters and enhancers. As a consequence, the IGS region may show a marked variation in length, due to the redundancy of those functional elements (repeats) $[5,6]$. Moreover, a higher number of duplicated regulatory elements has been related to an increased rate of rDNA transcription, which seems to play an adaptive role ([2] and references therein).

While the overall structural organization of the RNA-PolI machinery is comparable in all eukaryotes, the regulatory repeats are quite variable within each species, and hardly comparable between species, so that the region associated with the rDNA transcription promoter is generally species-specific (i.e., the RNA-PolI complexes of one species fail to transcribe the rRNA genes of another) ([7] and references therein). Consequently, IGS and RNA-PolI transcription factors seem to co-evolve in a species-specific way; therefore, this appears as a good candidate system for analyses on evolutionary co-adaptation of gene complexes [8]. As a matter of fact, selection must be taken into account to understand rDNA dynamics, so that, on the whole, the evolution of rDNA clusters is likely under a balanced influence of selection and "concerted evolution". In more detail, selection likely acts differently, the coding regions being more likely under purifying selection, whereas the IGS may experience more relaxed selective pressure [4].

Then, the repeated organization of rDNA would allow the spreading of new variations trough the mechanisms of genomic turnover, as well as the sexual reproduction could allow to fix them in the reproductive units (i.e. "concerted evolution"). In this scenario, any mutation in the controlling region, which would produce a better interaction to an essential transcription factor, should be selected for and fixed by unequal crossing over and gene conversion events during gametogenesis, as well as in somatic cells trough mitotic recombination [9]. As a consequence of those mechanisms, the rDNA clusters, as well as their dedicated transcription machinery, would experience rapid evolutionary changes, which could lead to a rapid evolution of post-mating isolation mechanisms. All that considered, the rDNA cluster represents a good candidate for studies on speciation. With this in mind, we started a characterization of the rDNA cluster of the Mediterranean genus Bacillus, which comprises two bisexual species (Bacillus grandii and Bacillus rossius), three unisexual parthenogenetic species (Bacillus atticus, Bacillus whitei and Bacillus lynceorum), and two hybridogenetic strains (Bacillus rossius-grandii benazzii and Bacillus rossius-grandii grandii). On the whole, Bacillus taxa constitute a clear case of reticulate evolution (i.e. the exploitation of a wide array of reproductive modes leading to a net of phyletic links) and provide a good experimental system to analyze the multifaceted relationships among sexual and metasexual taxa $[10,11]$.

This paper deals with the description of the IGS-ETS in $B$. atticus and B. grandii, two closely related species, as evidenced by mitochondrial and satellite DNA analyses 
$([10,11]$ and references therein). B. grandii - a strict bisexual taxon - shows a relic disjunct distribution in Sicily, and is divided into three subspecies, $B$. grandii grandii, $B$. grandii benazzi and B. grandii maretimi. Unlike B. grandii, $B$. atticus - at present an all-female obligate parthenogen - shows a much wider distribution in the western Mediterranean basin, and forms a complex of three different karyological $(2 n=32 ; 2 n=34 ; 3 n=48-51)$ and allozymic races, without morphological diversification. Based on this, $B$. atticus has been divided into three subspecies, namely B. atticus atticus, B. atticus carius and B. atticus cyprius $[10,11]$.

The characterization of Bacillus IGS-ETS, the first known for a phasmid insect, allowed us to infer about the variability and evolution of this peculiar tandemly-repeated system, and to compare it to the additional IGS-ETSs known to date in arthropods. Moreover, patterns of evolution of rDNA repeated sequences in Bacillus could be easily comparable to what has been observed for Bacillus pericentromeric satellite DNA. Actually, the Bacillus system represents an intriguing model to investigate how "concerted evolution" works on repeated sequences, because the existence of either apomictic and automictic parthenogens provides a unique opportunity to study the effects of meiosis and sex in multigene family evolution $[10,11]$. In more detail, the automictic parthenogen $B$. atticus, although maintaining meiosis and crossing over, lacks chromosome reshuffling due to sexual reproduction: for this reason, and according to the "concerted evolution" rationale, new variants can easily spread within a single B. atticus female and her progeny (i.e. a clone of parthenogens), but cannot spread in the whole taxonomic unit. On the other hand, the bisexual $B$. grandii, being the closest relative of $B$. atticus, represents the perfect counter- part, since in this species the repeated sequences would follow the "concerted evolution".

\section{Methods \\ IGS characterization}

Specimens of Bacillus atticus, B. grandii grandii, B. g. benazzi and B. g. maretimi were collected at several Mediterranean locations. Taxa, localities and sample acronyms are reported in Table 1.

Field-collected specimens were stored at $-80^{\circ} \mathrm{C}$. Total genomic DNA was isolated from somatic tissues with a standard phenol-chloroform protocol.

Since we didn't have any specific PCR primer to amplify the whole rDNA cluster, we decided to proceed with a wide restriction enzyme screening using the following enzymes: Taq, Alu, Sma, Ava, HpaI, HpaII, ClaI, SstI, StuI, CfoI, HhaI, MspI, DraI, HincII, XbaI and PstI. Such exploratory restriction analysis was performed on specimens of B. atticus from Castel di Tusa (Italy), Neraida (Greece) and Israel. XbaI produced a ladder-like pattern (the shorter fragment at about $350 \mathrm{bp}$, here referred to as $\mathrm{XbaI}_{350}$ ), while PstI produced two main fragments, a small one of $530 \mathrm{bp}$ and a high molecular weight one of $5.5 \mathrm{~kb}$ (here referred to as, respectively, $P_{s t} I_{530}$ and $P s t I_{5500}$ ). The ladder-like pattern of the XbaI fragments evidenced that this sequence is tandemly repeated. Total genomic DNA from $B$. atticus and $B$. grandii specimens (see Table 1 ) were then digested using PstI in order to obtain $P_{s t} I_{530}$ and $P s t I_{5500}$ fragments.

Fragments were separated by agarose gel electrophoresis and transferred into a nylon membrane by Southern blotting. Two probes were used for hybridization: the first was the $\mathrm{XbaI}_{350}$ fragment, the latter was a PCR-obtained por-

Table I: rDNA characterization in Bacillus

\begin{tabular}{|c|c|c|c|c|}
\hline Taxon & locality & acromyms & GenBank A.N. & \\
\hline & & & Bag530-Pstl clones & 3'IGS-ETS \\
\hline \multirow[t]{3}{*}{ Bacillus atticus atticus ( $2 n=34, q$ $q)$} & Palaeochora, Greece & AAT/Pal & 4 [EU855066-EU855070] & [EU855050] \\
\hline & Castel di Tusa, Italy & AAT/Tus & 9 [EU85507I-EU855079] & [EU855049] \\
\hline & Golan, Israel & AAT/lsr & 12 [EU855054-EU855065] & [EU855048]* \\
\hline \multirow[t]{2}{*}{ Bacillus atticus cyprius $(2 n=32$, 웅) } & Episkopi, Cyprus & ACY/Epk3 & 4 [EU855090-EU855093] & \\
\hline & & ACY/Epk4 & 6 [EU855094-EU855099] & [EU855046] \\
\hline Bacillus atticus carius ( $3 n=51, q$ q $)$ & Neraida, Greece & $\mathrm{ACA} / \mathrm{Ner}$ & 10 [EU855080-EU855089] & [EU855047] \\
\hline \multirow[t]{3}{*}{ Bacillus grandii grandii $\left(2 n=34,+\bigcirc^{7}\right)$} & Cava Grande del Cassibile, Italy & GG/Cag28 & I [EU855|II] & - \\
\hline & & GG/Cagl37 & 2 [EU855।|2-EU855।|3] & [EU85505I] \\
\hline & & GG/Cagl39 & 4 [EU855।|4-EU855।17] & - \\
\hline Bacillus grandii benazzii $\left(2 n=34\right.$,,$\left.\sigma^{7}\right)$ & Torre Bennista, Italy & GB/Tbe4 & $6[\underline{E U 855105}-\underline{E U 855110}]$ & [EU855052] \\
\hline Bacillus grandii maretimi $(2 n=34$, & Marettimo Is., Italy & GM/Marl & 5 [EU855100-EU855104] & [EU855053] \\
\hline
\end{tabular}

Species, localities, sample acronyms and GenBank A.N. of the analyzed specimens.

우 $ᄋ=$ all female parthenogen; $ㅇ=$ bisexual;

$*=$ Clone obtained by sequencing the portion of Pst $\left.\right|_{5500}$ from the last Bag550 repeat to the $5^{\prime}$-end of the $18 \mathrm{~S}$ gene. 
tion of the $18 \mathrm{~S}$ gene (5'-end), amplified with the following universal primers: 18S-s22(F) 5'-TAATGATCCTTCCG CAGGTTCA-3'; 18S-A1984(R) 5'-TCCCTGGTTGATCCTGCCAGTA-3', using Herculase II fusion DNA polymerase according to manufacturer's instructions. The probes were digoxigenine-labeled and hybridization was performed at $65^{\circ} \mathrm{C}$ accordingly to DIG DNA Labeling and Detection Starter Kit I (Roche); filters were washed with low stringency buffer $(0.5 \times \mathrm{SSC}, 0.1 \% \mathrm{SDS})$, corresponding to $\sim 80 \%$ sequence similarity between probe and target.

The fragments $\mathrm{XbaI}_{350}, \mathrm{PstI}_{530}$ and $\mathrm{PstI}_{5500}$ from B. atticus from Israel (AAT/Isr) were cloned into a pGem-7Zf(+) plasmid (Promega) vector using the E. coli DH5 $\alpha$ competent cells (Invitrogen). 64 PstI $_{530}$ clones were also obtained by restriction from B. grandii and B. atticus specimens (see Tab. 1). Recombinant clones were screened by PCR using M13 universal primers. Some of the obtained recombinant colonies were purified using SNAP Miniprep kit (Invitrogen) according to the manufacturer's instructions.

Clones were sequenced using M13 universal primers by Macrogen Inc. The portion upstream the $18 \mathrm{~S}$ gene of the PstI $_{5500}$ fragment was sequenced using "primer walking" method in B. atticus from Israel (AAT/Isr) (Table 1), and this allowed us to determine the IGS structure in B. atticus.

The PstI $I_{530}$ fragment was also used as a probe for in situ hybridization according to [12] on B. atticus chromosomes.

Given the observed structure of the IGS-ETS (see Results), and to perform a large comparison of IGS-ETSs among Bacillus species with a reasonable sequencing effort, we decided not to sequence the whole PstI ${ }_{5500}$ fragment for all $B$. grandii and $B$. atticus specimens, but to focus our attention on the 3' portion of the IGS and the ETS, from the last $P_{s t} I_{530}$ repeat and the $5^{\prime}$ of the $18 \mathrm{~S}$ gene (a region here referred to as ${ }^{3}$ IGS-ETS). To obtain these fragments, the PCR was performed with Herculase (Stratagene) following the manufacturer's instructions. A primer (Bag $(\mathrm{F})-5^{-}-$ ACAGGCAAATGGGAGTTG-3') was designed on the Pst $I_{530}$ Consensus sequences. This primer was then coupled with the $18 \mathrm{~S}$ universal primer by [13], modified as follows 18i(R)-5'-TTTCTCAGGCTCCCTCTCCGGAATCGAAC-

CCT- 3 '. The PCR products were amplified and completely sequenced using the "primer walking" method as described above. Analyzed specimens and GenBank Accession numbers are reported in Table 1.

\section{Sequence analyses}

All sequences were aligned using the CLUSTAL algorithm of the Sequence Navigator Software (Applera) and corrected by eye. Phylogenetic analyses on PstI ${ }_{530}$ and 3'IGS-
ETS were performed using Neighbor-Joining (NJ), Maximum Parsimony (MP) and Maximum Likelihood (ML) using PAUP* v.4.0b [14]. Likelihood scores for each DNA substitution model were estimated using MODELTEST and used for subsequent analyses [15]. The best-scored model was $\mathrm{JC}+\Gamma$ for PstI $_{530}$ and $\mathrm{F} 81+\Gamma$ for IGS-ETS sequences. Support for each node was obtained using bootstrap (500 replicates) [16]. Jukes-Cantor nucleotide distance values and nucleotide composition were calculated with MEGA 4.0 [17]. Nucleotide diversity (Pi) distribution across Pst $_{530}$ clones and ${ }^{3}$ IGS-ETS were calculated with a 30 and $60 \mathrm{bp}$ sliding window and step size of 15 and 10 bp respectively, using DnaSP program v.4.1 [18]. The conserved and variable regions were considered significant when Pi exceed $2 \times$ SD (standard deviation) the average $\mathrm{Pi}$, as described in [19].

Secondary structures were analyzed with the MFOLD server program [20]. To search for the presence of any internal repeat, the ${ }^{3}$ IGS-ETS sequences were analyzed using the server program REPFIND [21]. The Neural Network Promoter Prediction tool [22] was used to identify potential promoter sequences.

\section{Results}

Endonuclease restriction analysis of Bacillus atticus showed restriction fragments for XbaI and PstI: XbaI produced a ladder-like pattern, with the shorter band at about $350 \mathrm{bp},\left(\mathrm{XbaI}_{350}\right)$, which indicates the presence of a tandemly repeated DNA. PstI produced two main fragments, one of about $530 \mathrm{bp}\left(\operatorname{PstI}_{530}\right)$, the other at about $5.5 \mathrm{~kb}$ (PstI 5500$)$.

Southern blot analysis (Fig. 1) and sequencing unequivocally revealed that $\mathrm{XbaI}_{350}$ is part of the PstI $I_{530}$ sequence, henceforth named Bag530.

Southern blot analysis also evidenced that the $P s t I_{5500}$ fragment is part of the ribosomal DNA, containing both sequences of the $18 \mathrm{~S}$ gene and Bag530 (i.e. both $\mathrm{XbaI}_{350} /$ Pst $I_{530}$ fragments)(Figure 1). Direct sequencing evidenced that the PstI ${ }_{5500}$ fragment in B. atticus from Israel includes part of the last Bag530 monomer at the 5 ' end, while at the 3 'ends downstream the $18 \mathrm{~S}$ gene, therefore including the full ETS sequence (Figure 2). Moreover, in situ hybridization using $P_{s t} I_{530}$ as a probe evidenced that it localizes in the NOR of B. atticus (data not shown). Bag530 and 3'IGSETS fragments were then sequenced in $B$. atticus and $B$. grandii subspecies, as reported in Table 1.

\section{Structural organization of the IGS-ETS in Bacillus}

In $B$. atticus and $B$. grandii, the average nucleotide content of $3^{\prime}$ IGS-ETS sequences is $52.9 \% \mathrm{~A}+\mathrm{T}$ (range $52.5 \%$ $53.8 \%$ ), while the 374 bp belonging to the 5 -end of the structural $18 \mathrm{~S}$ gene show $53.1 \% \mathrm{~A}+\mathrm{T}$ content. On the 


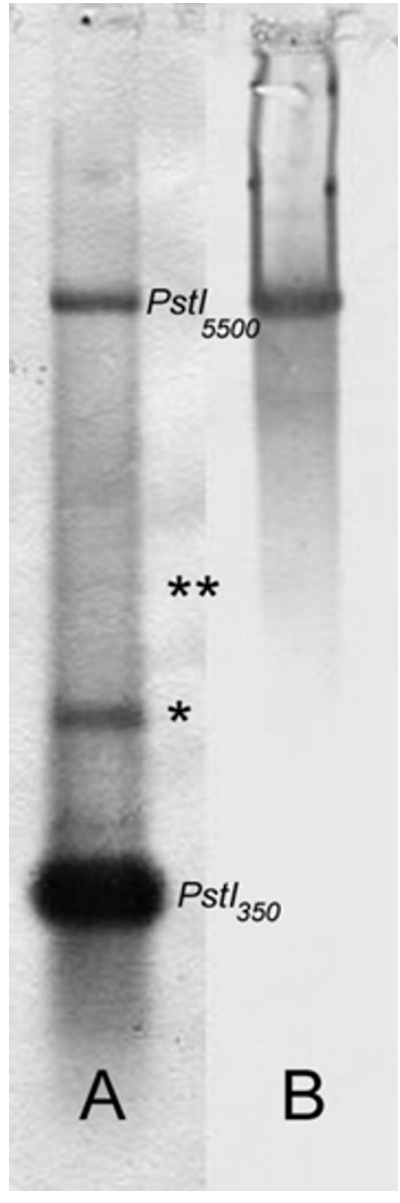

\section{Figure I}

Southern blot. Southern blot of the Pstl restriction pattern in B. atticus, using $\mathrm{Xbal}_{350}(\mathrm{~A})$ and the PCR-obtained 5 '-end of the $18 \mathrm{~S}$ gene $(B)$ as hybridizing probes. Southern Blot analysis revealed that: i) $\mathrm{Xbal}_{350}$ and Pstl $_{530}$ cross-hybridize, with $\mathrm{Xbal}_{350}$ being part of the Pst ${ }_{530}$ fragment (also tested by subsequent sequencing); ii) $\left.P s t\right|_{530}$ is a tandemly repeated sequence $\left(*=\right.$ Pst $_{530}$ dimer; $* *=$ Pst $_{530}$ trimer), as also supported by $\mathrm{Xbal}_{350}$ restriction pattern (not shown); iii) I8S PCR probe and $\mathrm{Xbal}_{350}$ hybridize with $\mathrm{Pstl}_{5500}$, so they are associated; iv) the $P s t I_{5500}$ band is the rDNA cluster, since it hybridizes to the I8S probe (also checked by subsequent sequencing).

whole the average length of the 3'IGS-ETS is $2583 \mathrm{bp}$, including $347 \mathrm{bp}$ of the $18 \mathrm{~S}$ gene (see additional file 1 material for ${ }^{3}$ IGS-ETS alignment and annotation).

Figure 2 summarizes the structure of the IGS-ETS region in Bacillus, as determined by sequencing the Pst $_{5500}$ and 3'IGS-ETS fragments. The exact position of 5' end of $18 \mathrm{~S}$ was established by aligning Bacillus sequence to the $18 \mathrm{~S}$ sequence of Blattella germanica [GenBank:AF005243]. The
IGS-ETS region showed the typical basic structure with both repetitive arrays and non-repetitive sequences. Repetitive regions are of two main types: a large cluster of head-to-tail repeats, corresponding to the Bag530 array, and a twofold 388 bp direct repeat (named Bag388a and Bag388b respectively) spaced by a 191 bp unique sequence. From the 3'IGS-ETS alignment (see additional file 2 data) it is evident that the last monomer of the Bag530 is differently truncated, namely at the position 280 in B. atticus and at the 260 in B. grandii. The first unique sequence ( $590 \mathrm{bp}$ long) characterizes the 5 ' of the IGS-ETS, downstream the Bag530 cluster. The Bag388a repeat is located in the position $871 / 1258$, while the Bag388b is between 1446/1835. The two Bag388a and $B a g 388 b$ repeats are separated by a sequence of 191 bp and followed by a unique sequence (428 bp long) upstream the 5 ' end of $18 \mathrm{~S}$.

Although the boundary between the IGS and ETS has not being actually determined, we found a motif similar to the transcription start point (tsp) sequence of other arthropods, downstream the Bag530 cluster. The observed motif (5'-TATATTAGAGGGA-3') well matches to the promoter consensus sequence (5'-TATA>TANGRRRR-3') of several arthropods (see additional file 3 data; [23]), and it has been also confirmed by using the Neural Network Promoter Prediction tool, which predicted the same sequence (5'-TTTTGGGTATATTAGAGGGA-3') with a score of 0.93 . Assuming that we did find the real gene promoter, ETS is therefore 1736 bp long in B. atticus and 1727 bp long in $B$. grandii. This length is quite different from what typically found in other arthropods, where the ETS regions are usually 500-1000 bp long [2], with few exceptions such as Daphnia pulex (1280 bp) [23].

\section{The Bag530 repeat}

As evidenced by restriction analysis and sequencing, the IGS region of Bacillus is characterized by an arrays of headto-tail tandemly-repeated Bag530 repeats. In this study we sequenced 64 Bag530 repeats obtained from restriction analysis from $B$. grandii and $B$. atticus (see Table 1 for details). Bag530 consensus sequence of 531 bp was obtained by aligning the 64 sequences; we observed a total of 42 indels, 11 of which representing a large deletion in all B. grandii grandii clones (see additional file 3 data). Average sequence length is $516 \mathrm{bp}$, with variants ranging from $505 \mathrm{bp}$ (GG/Cag137-c2) to 520 bp (GB/ Tbe4-c4 and c5).

Closer sequence examination revealed that Bag530 repeats could be further divided into three shorter subunits of 119 bp (A), 276 bp (B) and 120 (A'), respectively: $A$ and $A^{\prime}$ subunits showed $73.2 \%$ of sequence similarity between them. Due to the tandemly-arranged structure of Bag530, and to the fact that repeat boundaries are usually 


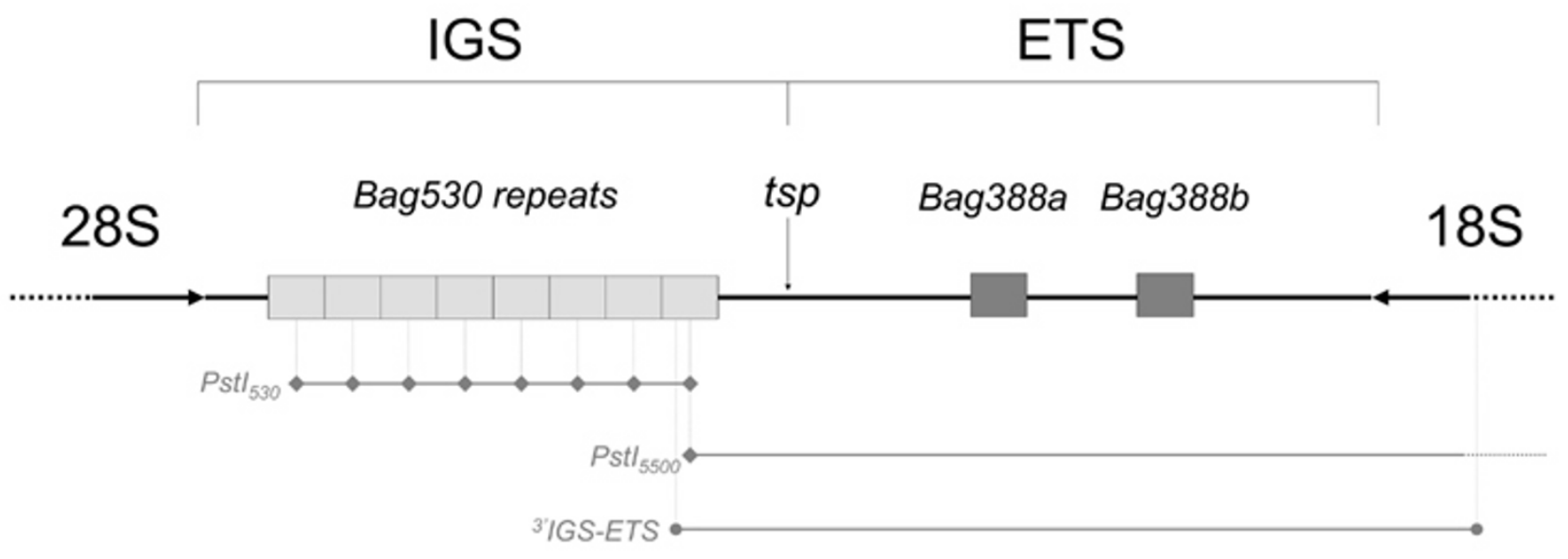

\section{Figure 2}

The IGS-ETS of Bacillus stick insects. Schematic drawing of the IGS-ETS structure of Bacillus stick insects. The IGS region is characterized by a large array of tandemly repeated head-to-tail sequences, here named Bag530, that are proposed to be RNA-Poll enhancers. Downstream the last Bag530 sequence, the transcription start point (tsp) mark the IGS-ETS boundary. Redundant tsp sequences are also found in each Bag530 enhancer. The ETS show a twofold 388 bp direct repeats (Bag388a and Bag388b), with an unknown regulatory function. Sequenced fragments are reported in gray: fragments obtained by Pstl restriction analysis $\left(\left.P_{s t}\right|_{530}\right.$ and $\left.\left.P s t\right|_{5500}\right)$ are marked with gray rhombs $(\bullet) ;{ }^{\prime}$ IGS-ETS fragment, obtained by PCR, is marked with gray circles (O).

defined arbitrarily by the restriction site, we could argue that the functional sequence of $B a g 530$ subrepeats should actually be $B-A$ '-A, because the last Bag530 monomer of the array appears to be truncated just downstream the A subrepeat (see above). Moreover, a putative promoter sequence (5'-TATATAGGGGGT-3') occurs in each of the Bag530 repeats, suggesting that these sequences are actually spacer promoters. This hypothesis is also supported by the presence of a palindromic motif of $28 \mathrm{bp}$ in each repeat (5'-CCCGGCGATCGAGGCCTCGATCGCCGGG$\left.3^{\prime}\right), 88$ bp upstream the putative spacer promoter sequence. In fact, the local fold symmetry created by the palindrome is thought to provide the binding site for DNA-binding proteins that are often dimeric, like the UBF factor involved in the machinery of the RNA-PolI [24].

\section{Variability of ${ }^{3}$ 'IGS-ETS and Bag530 sequences}

In the automictic parthenogen B. atticus the overall mean distances value of ${ }^{3}$ IGS-ETS is $0.010 \pm 0.002$. The distance values among the distinct allozymic and karyological races (see Background) show the same order of magnitude when comparing B. atticus cyprius vs. B. atticus carius (0.016 $\pm 0.003)$ and B. atticus cyprius vs. B. atticus atticus $(0.012 \pm$ 0.002 ), while between $B$. atticus atticus and $B$. atticus carius the value is lower $(0.007 \pm 0.001)$. On the other hand, the bisexual $B$. grandii shows a nearly 6 -fold higher overall ${ }^{3}$ IGS-ETS distance value $(0.058 \pm 0.005)$ when compared to the unisexual $B$. atticus. Comparing the different $B$. grandii subspecies among themselves, the distance values range from $0.039 \pm 0.004$ (B. grandii maretimi vs. B. grandii benazzi) to $0.083 \pm 0.005$ (B. grandii benazzi vs. B. grandii grandii).

Variability is not evenly distributed along the ${ }^{3}$ IGS-ETS sequence: the sliding window analysis evidenced that nucleotide diversity progressively drops when approaching the $18 \mathrm{~S}$ gene, which is likely due to selective sweep (Figure 3A). Moreover, where the putative tsp gene promoter was located, the only observed variation involves a single substitution $(G>A)$ in $B$. grandii benazzii sample (5'-TATATTAGAAGG-3'). The observed high level of sequence conservation in this region gives further evidence about its structural and functional role for transcription.

The pattern of overall variability of $B a g 530$ evidenced in $B$. atticus unisexuals is considerably lower to that of the sexual B. grandii $(0.018 \pm 0.003$ and $0.045 \pm 0.006$, respectively). The values of variability between $B$. atticus subspecies are: $B$. atticus cyprius vs.B. atticus carius $(0.022 \pm$ $0.005)$, B. atticus cyprius vs. B. atticus atticus $(0.018 \pm$ $0.003), B$. atticus atticus and B. atticus carius $(0.019 \pm$ 0.003 ). Comparing the $B$. grandii subspecies, the distance values are: B. grandii maretimi vs. B. grandii benazzi $(0.021$ $\pm 0.005), B$. grandii benazzi vs. B. grandii grandii $(0.072 \pm$ $0.011)$, and $B$. grandii grandii vs.B. grandii maretimi $(0.078$ \pm 0.012 ). 
A

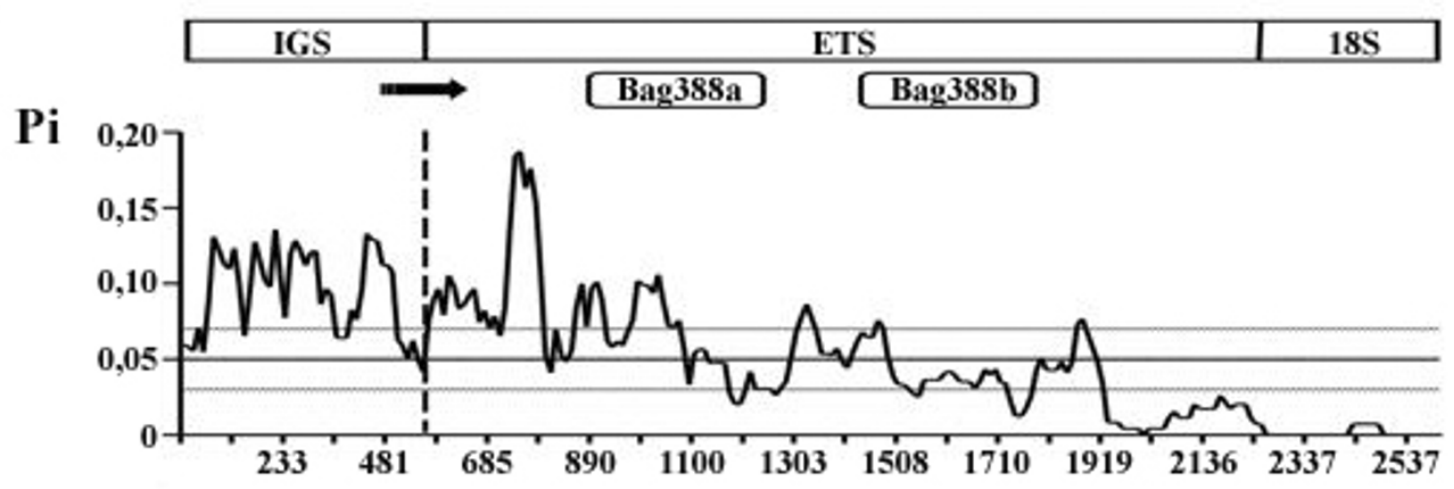

B

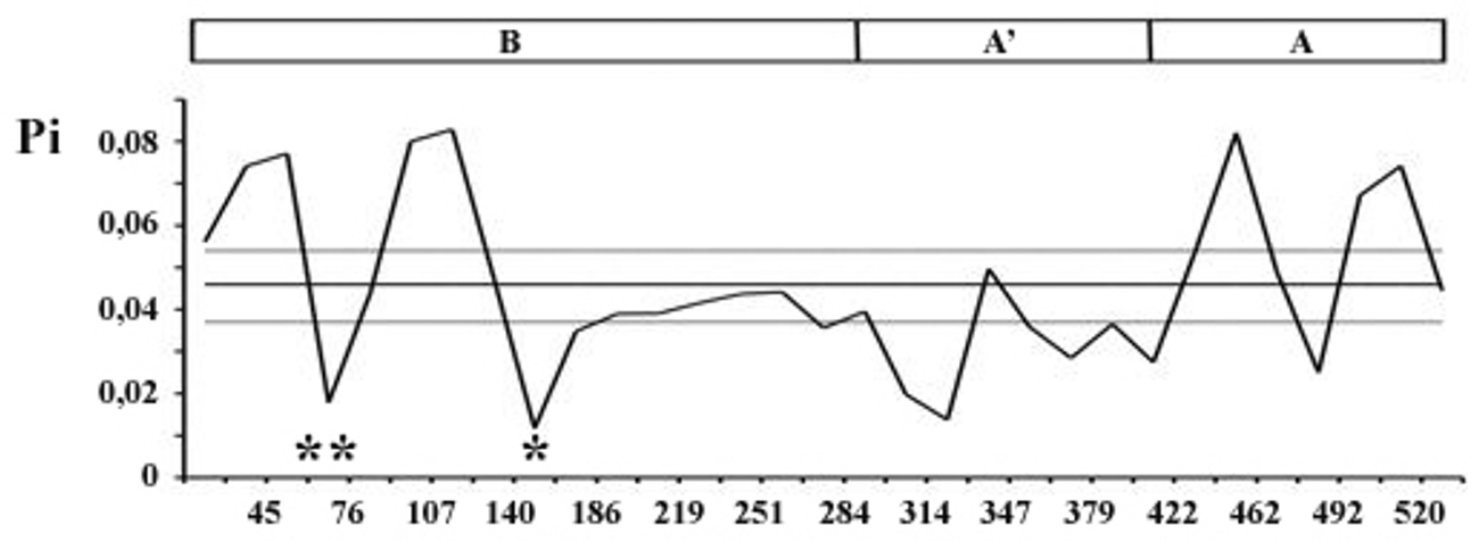

Nucleotide positions

\section{Figure 3}

Nucleotide variability distribution. A) Distribution of nucleotide variability along the ${ }^{3}$ IGS-ETS sequences in Bacillus by sliding window analysis (window size, 60 bp; step size, 10). The vertical dashed line indicates the IGS/ETS boundary. Above, the structure of ${ }^{3}$ IGS-ETS sequence and schematic drawing of functional elements are reported: arrow and solid blocks indicate putative gene promoter and Bag388, respectively. B) Distribution of nucleotide variability along the Bag530 monomers using sliding window analysis (window size, $30 \mathrm{bp}$; step size, 15). One $\left(^{*}\right)$ and two $\left(^{* *}\right)$ stars mark the position of the putative promoter and the palindromic sequences, respectively, which occur in each of the Bag530 repeats. Above, the Bag530 monomer structure has been represented. In both $A$ and $B$ sections, nucleotide positions (midpoint of sliding window) and nucleotide diversity are reported on the $X$ and $Y$ axes, respectively; average variability of $\mathrm{Pi}$ is shown by a solid line, dashed lines represent average variability \pm 2 SD (standard deviation).

An homogeneous range of variability was found within the populations of $B$. atticus atticus (Paleochora, $0.013 \pm$ 0.004; Castel di Tusa, $0.012 \pm 0.003$ and Israel, $0.015 \pm$ 0.003 ) and B. atticus carius (Neraida, $0.009 \pm 0.003$ ) and $B$. atticus cyprius (Episkopi, $0.009 \pm 0.002$ ). Also within $B$. grandii grandii and $B$. grandii benazzi,Bag530 has similar levels of variability $(0.010 \pm 0.003$ and $0.007 \pm 0.002$, respectively), while $B$. grandii maretimi showed a distance value equal to $0.015 \pm 0.003$, so that this subspecies seems at the first glance to be more variable than the others. However, it should be noted that such higher value is mainly due to a single Bag530 clone, only GM/Mar1-c3 (see Discussion): excluding it from the analysis, the level of variability falls to the value observed for $B$. grandii grandii and B. grandii benazzi $(0.010 \pm 0.003)$. 
It is interesting to note that sequence variability is not uniformly distributed in Bag530: two minima fall within the $\mathrm{B}$ subunit, one in the region including the palindromic motif of 28 bp (see above), and the other in the region including the RNA-PolI promoter (Figure. 3B). Actually, the promoter-like sequence shows no variation among all clones, as it has been observed in other organisms. It has been supposed that this sequences have the potential to form strong secondary structures suggesting that the region may be under functional constraints.

\section{Phylogenetic analysis}

Neighbor Joining, Maximum Parsimony and Maximum Likelihood trees based either on Bag530 clones or ${ }^{3}$ IGSETS sequences showed the same basic topology, with clones/sequences of $B$. atticus and B. grandii falling into two major distinct clades, well supported by bootstrap values. Here, for brevity, we report only Maximum Likelihood trees.
Trees based on Bag530 sequences (Figure 4A) showed two major distinct clades: one is given by $B$. grandii sequences, the other including $B$. atticus. Within B. atticus, clones grouped into two well-supported clusters: the first includes Bag530 clones of specimens collected in the western part of the species range (B. atticus carius and B. atticus atticus from Italy and Greece), while the second includes clones from specimens living in the Eastern Mediterranean (B. atticus cyprius from Cyprus and B. atticus atticus from Israel). Within each of the two B. atticus clusters, clones are completely intermingled, without any hint of geographical or population trend.

The B. grandii clones fall into three distinct clades, one for each subspecies, with $B$. grandii benazzi and $B$. grandii maretimi being more related. A single clone obtained from B. grandii maretimi (GM/Mar1C3) shows a peculiar clustering, being more similar to $B$. grandii benazzii variants (Fig. 4A). By comparing clones from B. grandii benazzii and $B$. grandii maretimi, 12 diagnostic positions were rec-

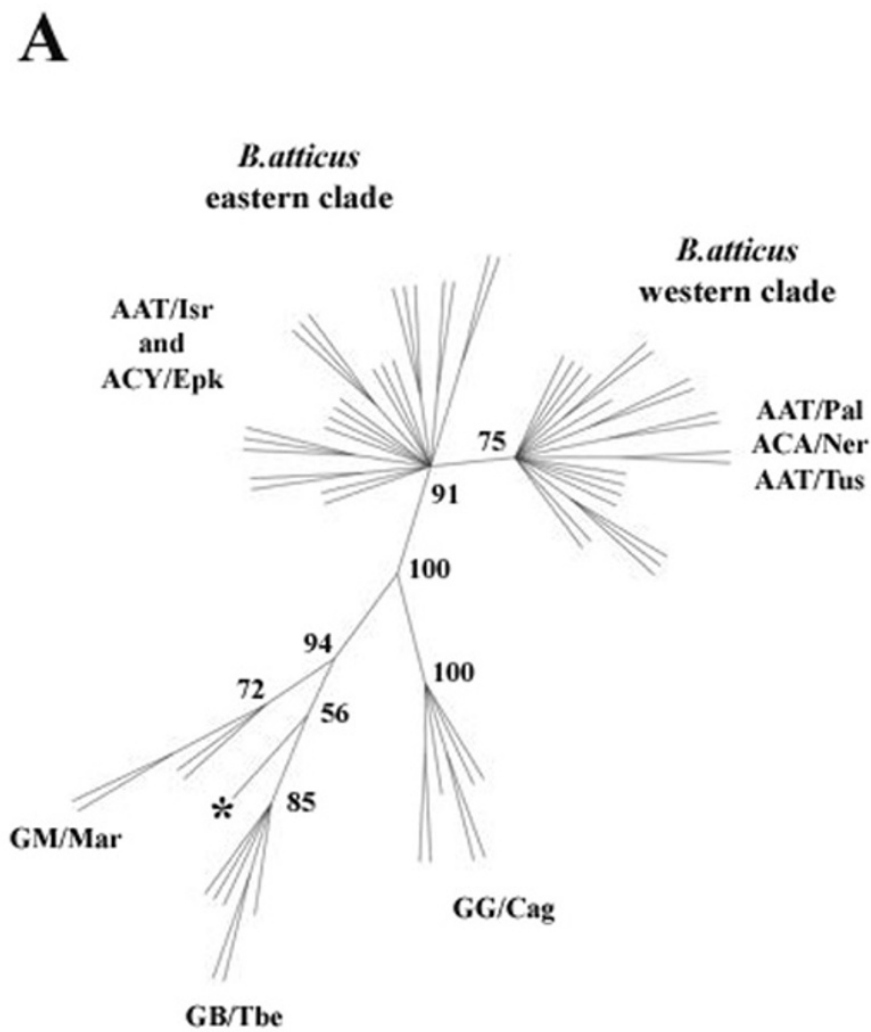

$-0.01$

\section{B}

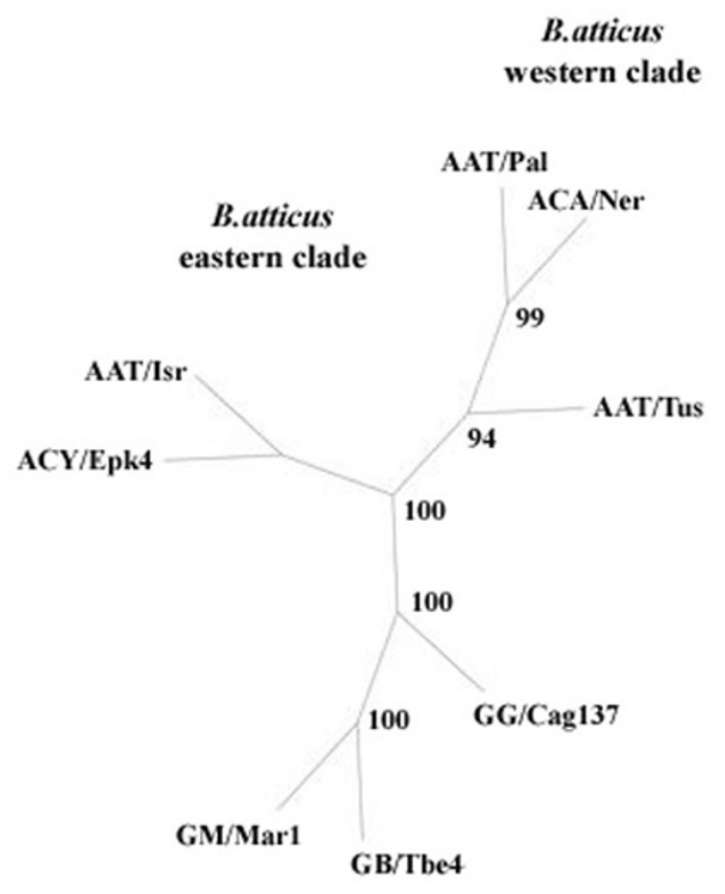

$-0.005$

\section{Figure 4}

Phylogenetic analysis. A) Maximum Likelihood unrooted cladogram obtained from Bag530 monomers. The recombinant clone GM/Mar I-c3 has been marked by a star (*). B) Maximum Likelihood unrooted cladogram obtained from 3'IGS-ETS sequences. Numbers at nodes are bootstrap values. The bars below the trees indicate mutational steps. 
ognized: GM/Mar1C3 shows the first nine diagnostic sites typical of $B$. grandii benazzii, while the last 3 are of $B$. grandii maretimi; therefore GM/Mar1C3 may be the result of a gene conversion.

The tree based on ${ }^{3}$ IGS-ETS sequences (Fig. 4B) shows the same basic topology as that based on Bag530 clones.

\section{Discussion \\ Structure of the IGS in Bacillus}

The molecular characterization of the IGS-ETS region showed that Bacillus atticus and B. grandii share the same basic structure and sequence (Fig. 2), with only minor variations; this result strongly supports the close phylogenetic relationship between the two species, as already indicated by previous mitochondrial and allozyme analyses ([10] and references therein).

As already mentioned, IGS-ETS sequences are fast evolving, and sequence homologies can be quickly lost; however, the observed structure of Bacillus IGS-ETS maintains several characteristics in common with other Metazoans. In detail, several organisms, such as Xenopus, Daphnia, mouse, rat, hamster, etc. ([5,6], and references therein) show tandem repeated sequence arrays downstream the 28S gene: for those the role of RNA-PolI transcription enhancers has been proposed [5]. Bacillus IGS shows repetitive elements of two main types: a large cluster corresponding to the Bag530 sequences and the twofold Bag388 direct repeat. Downstream the Bag530 cluster, a conserved putative promoter sequence has been found, which could represent the RNA-Poll promoter, and the same promoter is also present in each Bag530 repeat. Therefore we are quite confident in interpreting the Bag530 repeats as RNA-Poll enhancers.

Although no real sequence similarity could be detected, IGS enhancers must share the same mechanism of action, which likely is in their secondary structure; for instance, it has been observed that Xenopus enhancers work very well in mammals and in plants. Because of this common action, coupled with no obvious sequence similarity, enhancers could function as the binding sites of a common transcriptional factor, which most likely is the UBF (for a review see $[5,6]$, and references therein).

The presence of the Bag383a and Bag383b repeats in the ETS appears to be unique of Bacillus so, although they might have some sort of regulatory significance, nothing more precise can be stated at present.

Finally, the Bacillus rRNA at the junction between ETS and $18 \mathrm{~S}$ show the ability to form stem-loop structures, as observed in Drosophila melanogaster [25] and Apis mellifera [25], thus suggesting that this feature is likely important for an exact recognition of the rRNA splicing site. The stem-loop structure is formed by the conserved rRNA helices H9, H17 and H9' flanking the ETS region [26] (see additional file 4 material).

\section{Pattern of evolution of the IGS-ETS repeats and the "concerted evolution"}

The pattern of variability of Bag530 is quite different in $B$. atticus and $B$. grandii. In $B$. atticus some degree of geographic structure is observed, while $B$. grandii shows a more strong geographic and subspecies-specific clustering. This is also confirmed by the whole 3'IGS-ETS sequence analysis.

In more detail, a few Bag530 and 3'IGS-ETS variants are present in $B$. atticus, although, in theory, the absence of the homogenizing force of sexuality would permit the maintenance of many divergent variants in unisexuals. Moreover, Bag530 and ${ }^{3 '}$ IGS-ETS sequences evidenced the presence of two clusters in B. atticus, respectively including eastern (Cyprus and Israel) and western populations (Italy and Greece), regardless of subspecies, allozymic race or karyotype they belong to (see Table 1).

As a matter of fact, the presence of a slight geographic structuring of B. atticus Bag530 might be considered as an unexpected result, since, according to the "concerted evolution" rationale, the automictic $B$. atticus lacks chromosome reshuffling coupled with Mendelian reproduction, and this should prevent "spreading" and "fixation" of variants in the taxonomic unit. In Bacillus Bag320 satellite DNA the lack of either geographic or racial clustering was explained this way [27].

How can we explain this result for Bag530? First of all, it is relevant to remember that different kinds of selective pressures on tandemly repeated arrays produce different background in which molecular drive is fixing newly occurring variants. Although "concerted evolution" applies to IGSETS too, a substantial proportion of newly arisen variants may be eliminated by selection, because this region contains promoters and enhancers interacting with RNA-PolI machinery [28]. This would likely turn in the low variability observed, even in the absence of the homogenizing contribution of sexuality. Compared to rDNA, the satellite Bag320 family of Bacillus likely undergoes a more relaxed selection, so that unisexuals can maintain more variability in satellite DNA.

All that considered, we may speculate that the slight geographic structuring of B. atticus IGS-ETS might be an outcome of geographically-based co-evolution of the IGS-ETS RNA-PolI machinery. With this in mind, we still need to explain how newly arisen IGS-ETS variants might spread in a large geographical area, in absence of chromosome 
reshuffling due to sexual reproduction. We can suggest that populations carrying the new variant might have spread from a founding clone of $B$. atticus females, in which the new variant has been fixed by selection and genomic turnover mechanisms allowed by automictic parthenogenesis. However, another possibility must be mentioned, i.e. that by the time the new variant appeared, $B$. atticus was still a bisexual taxon. This hypothesis is not unrealistic, since it has been proved that in Sicily, at the time that the tri-hybrid B. lynceorum arose from B. rossius females (about 1 Myr ago), B. atticus males had to be present to form the hybrid [11]. At present, however, $B$. atticus males have never been observed in nature, although the species has been intensively collected.

In B. grandii, the pattern of variability of Bag530 and ' 'IGSETS might be explained by bisexuality acting as a driving force on repeated sequence homogenization/fixation in the same genomic pool (molecular drive), coupled with the co-evolution of the rDNA arrays within each gene pool. The result is a clear racial clustering of the Bag530 repeats, which is fully in line with what has been observed for the Bag320 Satellite DNA family [27]. Moreover, the invention of a recombinant clone between two Bag530 variants of $B$. grandii benazzii and $B$. grandii maretimi must be an outcome of the coexistence of both variants in the same genome, either now in the genome of $B$. grandii maretimi, or in a common ancestor of the subspecies, which are nowadays no longer sympatric. A very similar recombination event has been observed in the Bag320 satellite DNA of B. rossius [29].

Studies on satellite DNAs evidenced the occurrence of the so-called "library hypothesis" based on the "expansioncontraction" model: related taxa could share low-copy repetitive DNA sequences that can be specifically amplified in each evolutionary unit, thus becoming highly represented in one taxon while remaining at a low copy number in the others [30,31]. In agreement with the "library hypothesis", Mestrovic et al. [32] have recently proposed that rapid changes in satellite DNA profiles (within/between single family of satellite DNAs) could be achieved through replacements of functionally equivalent repeats of DNA, without affecting the DNA-proteins binding. The existence of specific profiles as a consequence of copy number changes in a set of satellite DNAs shared by related genomes was found in species of the insects Palorus and Pimelia [33,34],Bacillus [29] and in root-knot nematodes [32].

Therefore, the Bag530 GM/Mar1C3 clone might be in line with the "library hypothesis" of repeated DNA, since the observed recombination event should have occurred when both variants were present in the same genome; so we can speculate that rDNA enhancers, and likely the IGS-
ETS itself, might experience the same "expansion-contraction" mechanism described for other repeated DNAs.

\section{Conclusion}

All that considered, rDNA might be constrained by a complex array of evolutionary mechanisms: repeats homogenization achieved through genomic turnover mechanism may stabilize interactions with DNA-binding protein thus eliminating or limiting the effects of deleterious mutations (stable complex) but, simultaneously, the whole array could acquire new sequence variants, better fitting the above mentioned interactions (flexible system). The co-evolution can in turn be driven in a dual way, either by direct acquisition of newly mutated repeats, or by competition among repeats that better fit to the above mentioned DNA-proteins interactions. In this way, both DNAbinding proteins and repeat variants would drive each other's evolution leading to a new protein/DNA pair to replace the old one [35]. All this, coupled with sexuality might drive quick fixation of new rDNA variants in populations.

\section{Authors' contributions}

AR carried out the molecular genetic studies, sequence alignment, statistical analysis and drafted the manuscript. VS participated in the design of the study and helped drafting the manuscript. MP conceived of the study, participated in its design and coordination and helped to draft the manuscript. All authors have read and approved the final manuscript.

\section{Additional material}

\section{Additional file 1}

Sequence alignment of the ${ }^{3}$ IGS-ETS sequences in Bacillus. Sequence alignment and annotation of the ${ }^{3}$ IGS-ETS sequences in Bacillus. Species acronyms as in Table 1. Putative functional sequences are marked in bold. The stem and loop structure at the boundary between ETS and $18 S$ gene is reported above the sequence at the appropriate position of the alignment. Click here for file

[http://www.biomedcentral.com/content/supplementary/14712148-8-278-S1.pdf]

\section{Additional file 2}

Sequence alignment of the Bag530 sequences in Bacillus. Sequence alignment of the Bag530 sequences in Bacillus. Species acronyms as in Table 1. The " $C$ " followed by a number indicates the number of clone sequenced.

Click here for file

[http://www.biomedcentral.com/content/supplementary/14712148-8-278-S2.pdf] 


\section{Additional file 3 \\ Putative promoter sequences comparisons. Putative promoter sequence of $\mathrm{B}$. atticus and $\mathrm{B}$. grandii $(\mathrm{Ba}-\mathrm{g})$. Promoter sequences of Triops can- criformis (Tca), Daphnia pulex (Dpu) and Artemia franciscana (Afr) were also reported for comparison. The first nucleotide of the promoter sequence is indicated by (>) and seven bp of the upstream sequence are reported; $(g)$ and $(s)$ indicate gene and spacer promoters respectively. Consensus sequence of regions surrounding rDNA tsp of several arthro- pods is reported, as described by Crease (1993). \\ Click here for file \\ [http://www.biomedcentral.com/content/supplementary/1471- 2148-8-278-S3.jpeg] \\ Additional file 4 \\ Secondary structure of the ETS-18S junction. A) Secondary structure of the ETS-18S junction. Calculated free energy is reported below the struc- ture. B) Conserved rRNA helices (H9, H17 and H9') flanking 3'-end ETS are within dashed vertical line \\ Click here for file \\ [http://www.biomedcentral.com/content/supplementary/1471- 2148-8-278-S4.jpeg]}

\section{Acknowledgements}

We gratefully thank Prof. Barbara Mantovani, Dr. Andrea Luchetti and the two anonymous referees for their precious suggestions in manuscript revision. This work has been supported by the Italian "Ministero dell'Università e della Ricerca Scientifica" (MIUR) funds and by the "Donazione Canziani" bequest.

\section{References}

I. Eickbush TH, Eickbush D: Finely Orchestrated Movements: Evolution of the Ribosomal RNA Genes. Genetics 2007, I 75:477-485.

2. Burton RS, Metz EC, Flowers JM, Willett CS: Unusual structure of ribosomal DNA in the copepod Tigriopus californicus : intergenic spacer sequences lack internal subrepeats. Gene 2005, 344: $105-113$.

3. Dover GA: Molecular drive. Trends Genet 2002, I 8:587-589.

4. Weider LJ, Elser J, Crease TJ, Mateos M, Corner JB, Markow TA: THE FUNCTIONAL SIGNIFICANCE OF RIBOSOMAL (r)DNA VARIATION: Impacts on the Evolutionary Ecology of Organisms. Annu Rev Ecol Evol Syst 2005, 36:219-242.

5. Reeder RH: Regulation of RNA polymerase I transcription in yeast and vertebrates. Prog Nucleic Acid Res Mol Biol 1999, 62:293-327.

6. Moss T, Stefanovsky VY: Promotion and regulation of ribosomal transcription in eukaryotes by RNA polymerase I. Prog Nucleic Acid Res Mol Biol 1995, 50:25-66.

7. Heix J, Zomerdijk JCBM, Ravanpay A, Tjian R, Grummt I: Cloning of murine RNA polymerase I-specific TAF factors: conserved interactions between the subunits of the species-specific transcription initiation factor TIF-IB-SLI. Proc Natl Acad Sci USA 1997, 94:1733-1738.

8. Mian A, Dover GA: Promoter variation in the ribosomal RNA genes in Drosophila melanogaster strains. Nucleic Acids Res 1990, | 8:3795-380|.

9. Gantley ARD, Scott B: Extraordinary ribosomal length heterogeneity in a neotyphodium endophyte hybrid: implications for concerted evolution. Genetics 1998, I 50:1625-1637.

10. Mantovani B, Passamonti M, Scali V: The mitochondrial cytocrome oxidase II gene in Bacillus stick insect: ancestry of hybrids, androgenesis, and phylogenetic relationships. Mol Phylog Evol 200I, I9(I): I57-I63.

II. Scali V, Passamonti M, Marescalchi O, Mantovani B: Linkage between sexual and asexual lineages: genome evolution in Bacillus sticks insects. Biol J Linn Soc 2003, 79: I37-I50.
12. Marescalchi O, Scali V: Chromosomal and NOR patterns in the polyclonal stick insect Bacillus atticus atticus (Insecta Phasmatodea). Genome 1997, 40:261-270.

13. Hillis DM, Dixon MT: Ribosomal DNA: molecular evolution and phylogenetic inference. Quart Rev Biol 1991, 66:4 I I-453.

14. Swofford DL: PAUP* Phylogenetic Analysisi Using Parsimony (*and Other Methods), Version 4b. 200l [http:// paup.csit.fsu.edu/]. Sinauer Associates, Sunderland, Massachusetts

15. Posada D, Crandall KA: MODELTEST: testing the model of DNA substitution. Bioinformatics 1998, 14:817-818.

16. Felsenstein J: Confidence limits on phylogenies: an approach using the bootstrap. Evolution 1985, 39:783-79I.

17. Tamura K, Dudley J, Nei M, Kumar S: MEGA4: Molecular Evolutionary Genetics Analysis (MEGA) software version 4.0. Mol Biol Evol 2007, 24: I596-I599.

18. Rozas J, Sánchez-DelBarrio JC, Messegyer X, Rozas R: DnaSP, DNA polymorphism analyses by the coalescent and other methods. Bioinformatics 2003, 19:2496-2497.

19. Mravinac B, Plohl M, Ugarkovic D: Conserved patterns in the evolution of Tribolium satellite DNAs. Gene 2004, 332:I69-I77.

20. Zucker M: Mfold web server for nucleic acid folding and hybridization prediction. Nucleic Acids Res 2003, 3 I:3406-34 I 5.

21. REPFIND [http://zlab.bu.edu/repfind/]

22. Reese MG: Application of a time-delay neural network to promoter annotation in the Drosophila melanogaster genome. Comput Chem 200I, 26:51-56.

23. Crease TJ: Sequence of the intergenic spacer between the 285 and I 8S rRNA-encoding genes of the crustacean, Daphnia pulex. Gene 1993, I 34:245-249.

24. Arunkumar KP, Nagaraju J: Unusually long palindromes are abundant in mitochondrial control regions of insects and nematodes. PLOS ONE 2006, I:el I0.

25. Simeone A, La Volpe A, Boncinelli E: Nucleotide sequence of a complete ribosomal spacer of $D$. melanogaster. Nucleic Acids Res 1985, I3(4): 1089-I I01.

26. Gillespie JJ, Johnston JS, Cannone JJ, Gutell RR: Characteristics of the nuclear (I8S, 5.8S, $28 \mathrm{~S}$ and 5S) and mitochondrial (I $2 \mathrm{~S}$ and I6S) rRNA genes of Apis mellifera (Insecta: Hymenoptera): structure, organization, and retrotransposable elements. Insect Mol Biol 2006, I5(5):657-686.

27. Mantovani B, Tinti F, Bachmann L, Scali V: The Bag320 satellite DNA family in Bacillus stick insects (Phasmatodea): different rates of molecular evolution of highly repetitive DNA in bisexual and parthenogenetic taxa. Mol Biol Evol 1997, | 4(1 2): I | 97-1 205.

28. Nei M, Rooney AP: Concerted and birth-and-death evolution of multigene families. Ann Rev Genet 2005, 39: I2I - I52.

29. Cesari M, Luchetti A, Passamonti M, Scali V, Mantovani B: Polymerase chain reaction amplification of the Bag320 satellite family reveals the ancestral library and past gene conversion events in Bacillus rossius (Insecta Phasmatodea). Gene 2003, 3 1 2:289-295.

30. Fry K, Salser W: Nucleotide sequences of HS-alpha satellite DNA from kangaroo rat Dipodomys ordii and characterization of similar sequences in other rodents. Cell 1977, I 2:1069-1084.

31. Ugarkovic D, Plohl M: Variation in satellite DNA profiles-causes and effects. EMBO J 2002, 2 l:5955-5959.

32. Mestrovic N, Castagnone-Sereno P, Plohl M: Interplay of selective pressure and stochastic events directs evolution of the MELI 72 satellite DNA library in root-knot nematodes. Mol Biol Evol 2006, 23:2316-2325.

33. Mestrovic N, Plohl M, Mravinac B, Ugarkovic D: Evolution of satellite DNAs from the genus Palorus - Experimental evidence for the library hypothesis. Mol Biol Evol I998, I 5: I 062-1068.

34. Pons J, Bruvo B, Petitpierre E, Plohl M, Ugarkovic D, Juan C: Complex structural features of satellite DNA sequences in the genus Pimelia (Coleoptera: Tenebrionidae): random differential amplification from a common "satellite DNA library". Heredity 2004, 92:418-427.

35. Dawe RK, Henikoff S: Centromeres put epigenetics in the driver's seat. Trends Biochem Sci 2006, 31:662-669. 